\title{
Effect of Side Groups on the Hydrolytic Stability of Thiolated and Disulfide Cross-linked Polyaspartamides
}

\author{
Benjámin Gyarmati ${ }^{\star 1}$, Aysel Mammadova¹, Gergely Stankovits ${ }^{1}$, Dóra Barczikai ${ }^{1}$, András Szilágyi ${ }^{1}$ \\ ${ }^{1}$ Department of Physical Chemistry and Materials Science, Faculty of Chemical Technology and Biotechnology, Budapest \\ University of Technology and Economics, H-1521 Budapest, P.O.B. 91, Hungary \\ * Corresponding author, e-mail: bgyarmati@mail.bme.hu
}

Received: 18 July 2020, Accepted: 11 August 2020, Published online: 25 January 2021

\begin{abstract}
Thiolated polyaspartamides either with $N, N$-dimethyl-2-aminoethyl (DME), N,N-dimethyl-3-aminopropyl (DMP) or 2-hydroxyethyl (HE) side groups were synthesized to study the effect of side groups on the hydrolytic stability of these polymers. The chemical structure of linear polymers was confirmed by ${ }^{1} \mathrm{H}$ NMR spectroscopy, while thiol content was determined by Ellman's assay. Hydrolytic stability of thiolated polyaspartamides was studied by viscosity measurements and results suggested main-chain degradation in the presence of DME side groups. Polyaspartamides with DMP or HE side groups showed considerable stability. Thiolated polyaspartamides were converted to their disulfide cross-linked hydrogels and the gelation process was followed by oscillatory rheology. Time-dependence of dynamic moduli indicated the degradation of hydrogels containing DME side groups, while stable moduli were observed for hydrogels containing DMP or HE side groups. This structure-dependent aqueous degradation of polyaspartamides can be useful in different biomedical fields.
\end{abstract}

Keywords

polyaspartamides, rheology, hydrolytic stability, degradation

\section{Introduction}

Water-soluble, biodegradable polymers including poly(amino acid)s can be potentially used to deliver nucleic acids or other bioactive compounds into targeted cells without any toxic effect of the carrier or its degradation products [1-3]. Poly(aspartic acid) (PASP) as an anionic poly(amino acid) can be prepared by thermal polycondensation of L-aspartic acid to polysuccinimide (PSI), followed by a ring opening reaction in alkaline medium. The hydrophilic nature and biodegradable peptide linkage in the backbone made anionic PASP promising candidate in various applications, e.g. as anti-scaling agents or drug delivery vehicles [4-11]. Nevertheless, cationic polyaspartamides have been more extensively studied recently [12-14] as they can form electrostatic complexes with anionic biomolecules, e.g., nucleic acids and certain proteins $[15,16]$. For example, Sharma et al. [17] reported synthetic antimicrobial polyaspartamides with a biodegradable polyamide backbone and basic dimethylamine pendant groups, which were prepared by aminolysis of succinimide rings of PSI. Kravicz et al. [18] studied the role of

hydrophobic association on the gene transfection efficiency of cationic polyaspartamides and proved that the presence of tertiary amine groups is required for high effciency.

In addition to their definitive role in therapeutic uses, side groups also affect the degradation of polyaspartamides, which are prone to non-enzymatic, hydrolytic degradation through the imide formation of repeating units. Itaka et al. [19] showed the role of imide formation in the faster degradation of poly $\left\{N^{\prime}\right.$-[N-(2-aminoethyl)-2-aminoethyl]aspartamide]\} (PASP(DET)) as compared to the stability of its polyglutamide analogue (PGlu(DET)) in which the formation of a six-membered imide ring is not favored. Similarly, Lu et al. [20] studied the degradation of metal chelating polymers based on PGlu(DET) and PASP(DET) and a spontaneous main-chain degradation of PASP(DET) derivatives was observed within a few days, while PGlu(DET) was stable over two weeks. Neuse et al. [21] reported the effect of formation of intrachain type amide links on polymer stability and degradation, and proved the poor hydrolytic stability of polyaspartamides with an ethylene group between 
the main-chain nitrogen and the pendant nitrogen atoms. Although degradation of polyaspartamides have been studied by many researchers [19-23], there is still need for novel polymers with non-enzymatic, hydrolytic degradation to improve their potential in biomedical applications after careful mapping of the structure-property relationships.

We earlier reported the synthesis of polyaspartamides with tertiary amine side groups and proved that the type of side groups has a determining role in physicochemical properties including glass transition temperature and dissolution rate [24]. We also prepared PASP derivatives with pendant thiol groups which can be converted into disulfide linkages to prepare stable chemically crosslinked hydrogels [25]. Considering the literature data and our previous results, our hypothesis was that the combination of thiol and amine side groups enables us to prepare linear and cross-linked polyaspartamides with structure-dependent hydrolytic stability. Accordingly, in the current paper we report the synthesis of thiolated polyaspartamides with various additional side groups to study the effect of chemical structure on the possible degradation. Viscosity measurements were carried out on linear polyaspartamides to confirm any changes in molecular mass upon storage in aqueous solution. Oxidation-induced gelation and the stability of poly\{[(2-mercaptoethyl ) aspartamide $]$-co-[( $N, N$-dimethyl-2-aminoethyl)aspartamide $]\}$ (CYS20DME80), poly\{[(2-mercaptoethyl)aspartamide]-co-[(N,N-dimethyl-3-aminopropyl)aspartamide]\} (CYS20DMP80) and poly\{[(2-mercaptoethyl)aspartamide]-co- [(2-hydroxyethyl)aspartamide] (CYS20HE80) polyaspartamides were investigated by oscillatory rheology to determine the hydrolytic stability of such compositions. The change in dynamic moduli was used to study the gelation process [26] and the frequency dependence of the moduli was investigated to characterize the aqueous stability of the hydrogels. Finally, we aimed at revealing the effect of different side groups on the possible hydrolytic degradation of both linear and cross-linked polyaspartamides.

\section{Materials and methods \\ 2.1 Materials}

L-aspartic acid (99.5 \%), phosphoric acid (99\%), Ellman's reagent (5,5'-dithiobis(2-nitrobenzoic acid); $98 \%$ ), and monobasic potassium phosphate $(99.5 \%)$ were purchased from Merck. Deuterium oxide ( $\mathrm{D}_{2} \mathrm{O}, 99.9$ atom\% D, containing $0.05 \mathrm{wt} \%$ 3-(trimethylsilyl)propionic-2,2,3,3- $\mathrm{d}_{4}$ acid sodium salt) and 3-(dimethylamino)-propylamine
(DMP) (99 \%) were purchased from Sigma-Aldrich. $N, N$-dimethylethylenediamine (DME) and ethanolamine (HE) (99\%) was bought from Merck. Ethyl acetate (99\%) and acetone (99\%), citric acid (99\%) were purchased from Molar Chemicals. Cysteamine (CYS) (95\%), dibasic sodium phosphate monohydrate $\left(\mathrm{Na}_{2} \mathrm{HPO}_{4} \cdot \mathrm{H}_{2} \mathrm{O}\right)$, ethylenediaminetetraacetic acid disodium salt dihydrate $\left(\mathrm{Na}_{2}\right.$ EDTA $\left.2 \mathrm{H}_{2} \mathrm{O}\right)$, dithiothreitol (DTT; $99 \%$ ), hydrochloric acid $(\mathrm{HCl})$, monobasic sodium phosphate dihydrate $\left(\mathrm{NaH}_{2} \mathrm{PO}_{4} \cdot 2 \mathrm{H}_{2} \mathrm{O} ; 99 \%\right.$ ), N,N-dimethylformamide (DMF), sodium bromate $\left(\mathrm{NaBrO}_{3} ; 99 \%\right)$, sodium chloride $(\mathrm{NaCl})$, potassium chloride $(\mathrm{KCl})$, sodium hydroxide $(\mathrm{NaOH})$ were purchased from Reanal Hungary. All reagents were used without further purification and their quality was "for analysis" unless otherwise noted. Syntheses and measurements were carried out at $25^{\circ} \mathrm{C}$ unless otherwise noted. Ultrapure water ( $\rho>18.2 \mathrm{M} \Omega \mathrm{cm}$, Millipore) was used for the preparation of aqueous solutions.

Phosphate-buffered saline (PBS) solution of $\mathrm{pH}=7.7$ was prepared by dissolving $8.00 \mathrm{~g}$ of $\mathrm{NaCl}, 0.20 \mathrm{~g}$ of $\mathrm{KCl}, 1.44 \mathrm{~g}$ of $\mathrm{Na}_{2} \mathrm{HPO}_{4} \cdot 2 \mathrm{H}_{2} \mathrm{O}$ and $0.12 \mathrm{~g}$ of $\mathrm{KH}_{2} \mathrm{PO}_{4}$ in $1 \mathrm{~L}$ of water, the $\mathrm{pH}$ being adjusted with $0.1 \mathrm{~mol}$ $\mathrm{L}^{-1} \mathrm{HCl}$. Buffer solution of $\mathrm{pH}=5.5$ was prepared by dissolving $6.33 \mathrm{~g}$ citric acid (anhydrous) and $3.37 \mathrm{~g} \mathrm{KCl}$ in $83.73 \mathrm{ml}$ of $1 \mathrm{M} \mathrm{NaOH}$ and filled with water to reach $1 \mathrm{~L}$ volume. The phosphate buffer for Ellman's analysis $(\mathrm{pH}=8)$ was prepared by dissolving $5.33 \mathrm{~g}(34.2 \mathrm{mmol})$ of $\mathrm{NaH}_{2} \mathrm{PO}_{4} \cdot 2 \mathrm{H}_{2} \mathrm{O}, 82.92 \mathrm{~g}(465.8 \mathrm{mmol})$ of $\mathrm{Na}_{2} \mathrm{HPO}_{4} \cdot 2 \mathrm{H}_{2} \mathrm{O}$ and $0.37 \mathrm{~g}(1 \mathrm{mmol})$ of $\mathrm{Na}_{2}$ EDTA.2 $\mathrm{H}_{2} \mathrm{O}$ in $1 \mathrm{~L}$ of water. The $\mathrm{pH}$ of the buffer solutions was checked with a Mettler Toledo InLab®413 pH electrode.

\subsection{Synthesis}

Synthesis of polysuccinimide: Polysuccinimide was synthesized by the thermal polycondensation of aspartic acid as reported earlier [27]. The viscosity average molecular weight of the polymer was found to be $33 \mathrm{kDa}$ (determined by an Anton Paar Lovis 2000 rolling ball viscometer, Mark-Houwink constants were taken from the literature [28]: $K=1.32 \times 10^{-1} \mathrm{~g} / \mathrm{cm}^{3}$ and $\alpha=0.76$ ). Chemical structure of the polymer was confirmed by ${ }^{1} \mathrm{H}$ NMR (500 MHz, DMSO- $d_{6}, \delta(\mathrm{ppm}): 5.10(\mathrm{CH}) ; 3.20$ and $\left.2.75\left(\mathrm{CH}_{2}\right)\right)($ Scheme 1).

Synthesis of thiolated polysuccinimide: $1.00 \mathrm{~g}(0.0103$ mol succinimide (SI) repeating units) PSI was dissolved in $9.00 \mathrm{~g}$ DMF under nitrogen atmosphere in each case. To avoid premature oxidation, $0.1590 \mathrm{~g}(0.0010 \mathrm{~mol})$ dithiothreitol (DTT) (half of thiolated repeating units) was 
added to the mixture. $0.1587 \mathrm{~g}(0.0021 \mathrm{~mol})$ cysteamine (CYS) (20\% of SI repeating units) was added to the mixture after $10 \mathrm{~min}$ and stirred for additional $20 \mathrm{~min}$ under nitrogen atmosphere. To complete the reaction, the reaction mixtures were stirred for additional $72 \mathrm{~h}$ in sealed vials (Scheme 1). The same amount of thiolated PSI was used for each further modification.

Synthesis of poly\{[(2-mercaptoethyl)aspartamide] -co-[(N,N-dimethyl-2-aminoethyl)aspartamide]\} (CYS20DME80): $0.7270 \mathrm{~g} \quad(0.0082 \mathrm{~mol}) \quad N^{\prime} N-$ dimethylethylenediamine (DME) was added to the solution of thiolated PSI and stirred for $24 \mathrm{~h}$. CYS20DME80 was precipitated in ethyl acetate (the volume ratio of ethyl acetate to the polymer solution was 10:1), then washed three times with ethyl acetate and finally with acetone. The precipitate was then dissolved in $0.1 \mathrm{M}$ sulfuric acid (the molar ratio of sulfuric acid and the tertiary amine groups was 1:2) to convert the polymer into sulfate salts, dialyzed against water for one week (cellulose membrane, cut-off: $14000 \mathrm{Da}$ ) with daily change of water, concentrated in a rotary evaporator to around $10 \mathrm{ml}$ and freezedried to yield solid, off-white products (Scheme 1).

Synthesis of poly\{[(2-mercaptoethyl)aspartamide] -co-[(N,N-dimethyl-3-aminopropyl)aspartamide $]\}$ CYS20DMP80: 0.8427 g (0.0082 mol) 3-(dimethylamino)-propylamine (DMP) was added to the solution of thiolated PSI and stirred for $24 \mathrm{~h}$. CYS20DMP80 was precipitated in ethyl acetate (the volume ratio of ethyl acetate to the polymer solution was 10:1), then washed three times with ethyl acetate and finally with acetone. The precipitate was then dissolved in $0.1 \mathrm{M}$ sulfuric acid (the molar ratio of sulfuric acid to the tertiary amine groups was 1:2) to convert the polymer into sulfate salts, dialyzed against water for one week (cellulose membrane, cut-off: $14000 \mathrm{Da}$ ) with daily change of water, concentrated in a rotary evaporator ( $40^{\circ} \mathrm{C} ; 30 \mathrm{mbar}$ ) to around $10 \mathrm{ml}$ and freeze-dried to yield solid, off-white products (Scheme 1).

Synthesis of poly\{[(2-mercaptoethyl)aspartamide]-co[(2-hydroxyethyl)aspartamide] CYS20HE80: $0.5037 \mathrm{~g}$ $(0.0082 \mathrm{~mol})$ ethanolamine (HE) was added to the solution of thiolated PSI and stirred for $24 \mathrm{~h}$. CYS20HE80 was precipitated in ethyl acetate (the volume ratio of ethyl acetate to the polymer solution was 10:1), then washed three times with ethyl acetate and finally with acetone. The precipitate was then dissolved in water, dialyzed against water for one week (cellulose membrane, cut-off: $14000 \mathrm{Da}$ ) with daily change of water, concentrated in a rotary evaporator $\left(40^{\circ} \mathrm{C} ; 30 \mathrm{mbar}\right)$ to around $10 \mathrm{ml}$ and freeze-dried to yield solid, off-white products (Scheme 1).

\subsection{Chemical characterization}

The structure of polyaspartamides as well as degradation products was analyzed by using ${ }^{1} \mathrm{H}$ NMR spectroscopy (500 MHz, 16 scans). Solutions for the measurements were prepared by dissolving $20 \mathrm{mg}$ of freeze-dried polymer in $1 \mathrm{ml}$ of $\mathrm{D}_{2} \mathrm{O}$.

Ellman's assay was performed to determine the thiol content of polyaspartamides. $200 \mu \mathrm{L}$ of polymer solution (nominal thiol concentration was $0.5 \mathrm{mmol} / \mathrm{L}$ ) was diluted with $1600 \mu \mathrm{L}$ buffer solution, then $200 \mu \mathrm{L}$ of $1 \mathrm{mmol} / \mathrm{L}$ Ellman reagent was added to the solution. The reaction mixture was incubated at $37^{\circ} \mathrm{C}$ for 15 min during which the disulfide linkages of the reagent (5,5'-dithiobis-(2-nitrobenzoic acid)) reacts with the thiol groups of the polymers. The 2-nitro-5-thiobenzoate anions formed in equal amount as the thiols groups of the sample, which give a yellow

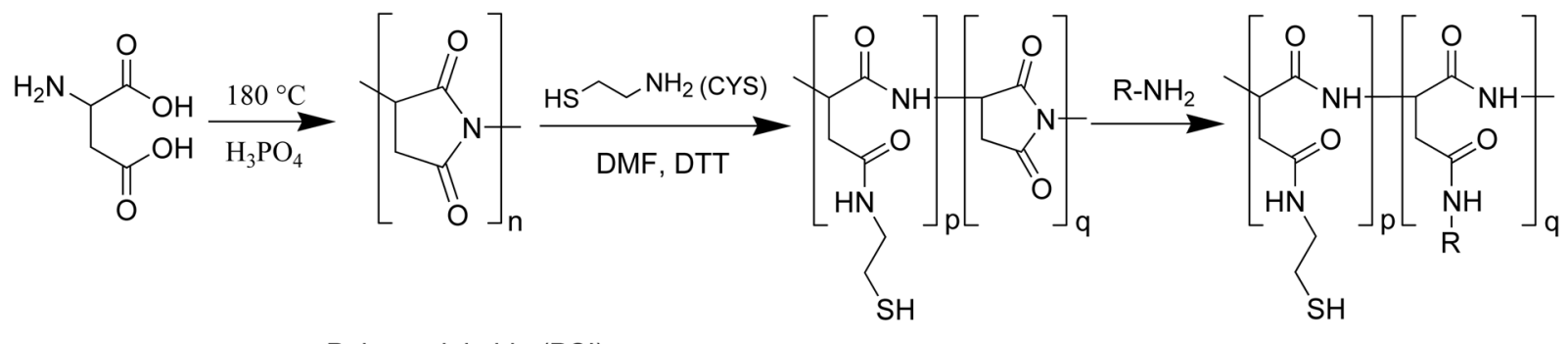

Polysuccinimide (PSI)

Thiolated polysuccinimide

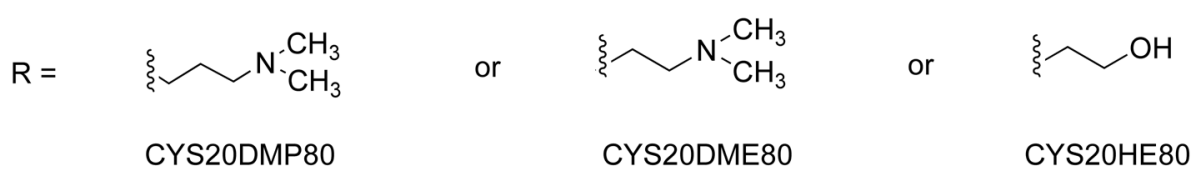

Scheme 1 Synthesis of CYS20DMP80, CYS20DME80 and CYS20HE80 
color of the solution $[25,29]$. Optical absorption spectra of the solutions between 300 and $800 \mathrm{~nm}$ were recorded by a Cary 60 UV-Vis Spectrometer (Agilent Technologies). Thiol content was calculated using the calibration determined over the thiol concentration range of $0.02-0.10 \mathrm{mmol} / \mathrm{L}$ (N-acetylcysteine, $\varepsilon=12.173 \mathrm{Lmol}^{-1} \mathrm{~cm}^{-1}$ ).

\subsection{Viscosity measurements}

Viscosity of aqueous solutions of CYS20DME80, CYS20DMP80 and CYS20HE80 polyaspartamides was determined immediately after preparation and after their storage at $\mathrm{pH}=7.7$ ). Each polymer was dissolved in water at a concentration of $2 \mathrm{mg} / \mathrm{ml}$ and the $\mathrm{pH}$ of the solution was adjusted to 7.7 by the addition of $\mathrm{NaOH}$ (buffer was not used for these measurements to eliminate the interfering effect of salts). The solutions were freeze-dried after $24 \mathrm{~h}$ and solid products were dissolved in a buffer solution at $\mathrm{pH}=5.5$ at a concentration of $10 \mathrm{mg} / \mathrm{ml}$. DTT was added in a concentration of $10 \mathrm{mM}$ to keep the polymers in their reduced state. Viscosity of the polymer solutions was measured in rolling ball viscometer (Anton Paar Lovis 2000 rolling ball viscometer, $\eta_{\text {solvent }}=1.00 \mathrm{mPas}$ ). The viscosity of the original polymers was also measured under the same conditions.

\subsection{Rheological tests}

Rheological measurements were carried out an Anton Paar Physica MCR301 rheometer with cone-plate geometry (CP-25-1; cone angle: $1^{\circ}$; sample gap: $0.054 \mathrm{~mm}$; $T=20^{\circ} \mathrm{C}$ ) in oscillatory mode. Polymer solutions were prepared by dissolving $10 \mathrm{mg}$ of each polymer in $90 \mathrm{mg}$ of PBS $(\mathrm{pH}=7.7)$ and $6 \mu \mathrm{L}$ of oxidant $\left(1 \mathrm{M} \mathrm{NaBrO}_{3}\right.$, $\left.n_{\mathrm{ox}} / n_{\mathrm{SH}}=1 / 2\right)$ was added to the polymer solution on the plate of the rheometer. The change of dynamic moduli was followed at constant strain $(\gamma=1 \%)$ and angular frequency $\left(\omega=1 \mathrm{rad} \mathrm{s}^{-1}\right)$ during gelation $(20 \mathrm{~min})$. After gelation, the frequency dependence of the moduli $(\gamma=1 \%$, $\omega=0.5-500 \mathrm{rad} \mathrm{s}^{-1}$ ) were recorded and this step was repeated in every hour for additional $18 \mathrm{~h}$. An excess amount of buffer solution was used on the lower plate to avoid evaporation.

\section{Results and discussion}

Thiolated polyaspartamides bearing a cationic side group ( $N, N$-dimethyl-2-aminoethyl (DME) or $N, N$-dimethyl3-aminopropyl (DMP) group) or a neutral side group (2-hydroxyethyl (HE) group) were synthesized. Degree of modification for each side group was determined by quantitative analysis of ${ }^{1} \mathrm{H}$ NMR spectra (Fig. 1) and thiol content was calculated from Ellman's assay.

The structural analysis of the synthesized
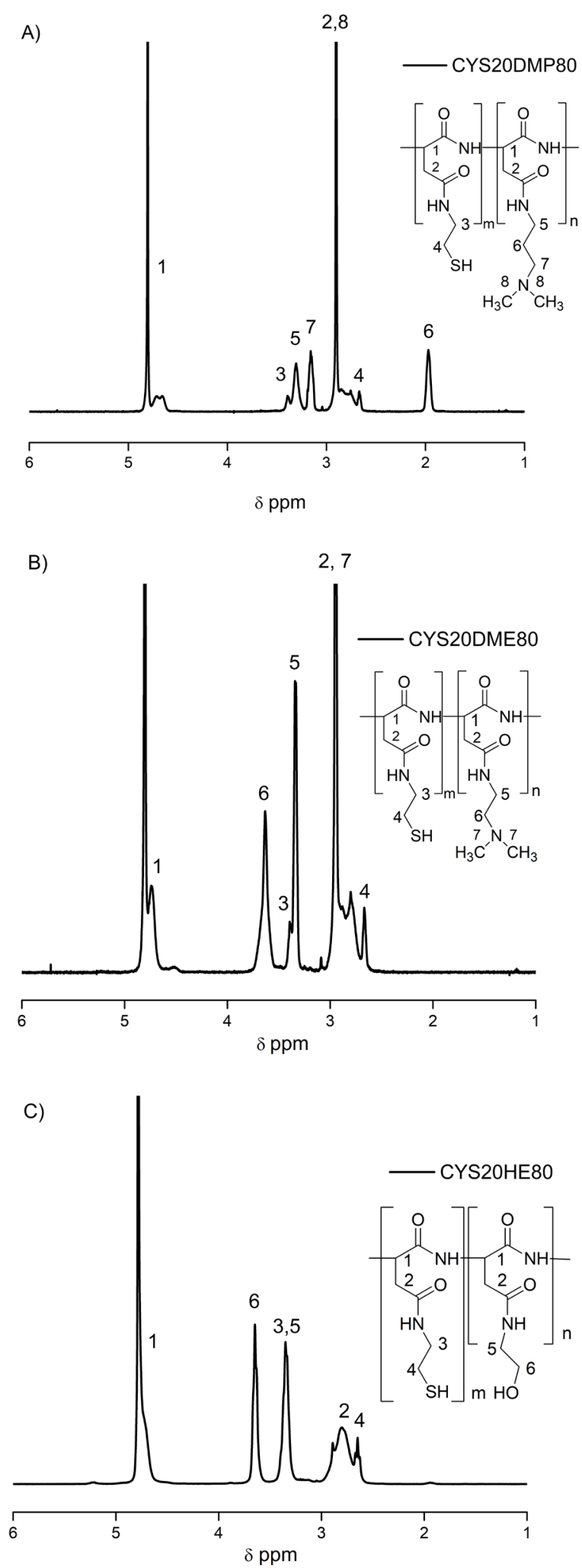

Fig. 1 1H NMR spectra of (a) CYS20DMP80, (b) CYS20DME80 and (c) CYS20HE80 
polyaspartamides was carried out in $\mathrm{D}_{2} \mathrm{O}$ by ${ }^{1} \mathrm{H}$ NMR. In Fig. 1(a) the broad signal at 2.00-1.90 ppm and 3.20$3.08 \mathrm{ppm}$ in CYS20DMP80 spectrum was correlated to the $2 \mathrm{H}$ protons of DMP side group in $\beta$ position (6) and $2 \mathrm{H}$ protons of DMP in $\gamma$ position to the amide group (7), respectively. Protons at 3.00-2.60 ppm was assigned to $2 \mathrm{H}$ protons of methylene group in backbone (2), $2 \mathrm{H}$ protons of CYS units in $\beta$ position to amide group (4) and $6 \mathrm{H}$ protons of DMP methyl groups (8). The $2 \mathrm{H}$ protons of CYS and $2 \mathrm{H}$ protons of DMP units in $\alpha$ position to amide groups $(3,5)$ give signal at 3.45-3.22 ppm range. The peak at $4.7 \mathrm{ppm}$ was correlated $1 \mathrm{H}$ proton of methyne group in the backbone (1).

In Fig. 1(b) the broad signal at 3.00-2.60 ppm in CYS20DME80 spectrum was correlated to the $2 \mathrm{H}$ protons of methylene group in backbone (2), $6 \mathrm{H}$ protons of DME methyl groups (7) and $2 \mathrm{H}$ protons of CYS units in $\beta$ position to amide group (4). Protons around 3.45-3.25 ppm was assigned to both $2 \mathrm{H}$ protons of CYS and DME units in $\alpha$ position to amide groups $(3,5)$, while protons at $3.80-$ $3.55 \mathrm{ppm}$ related to $2 \mathrm{H}$ protons of DME in $\beta$ position to amide group (6). The signal at $4.7 \mathrm{ppm}$ was correlated $1 \mathrm{H}$ proton of methyne group in the backbone (1).

In Fig. 1(c) the broad signal at 3.00-2.55 ppm in CYS20HE80 spectrum was correlated to the $2 \mathrm{H}$ protons of methylene group in backbone (2) and $2 \mathrm{H}$ protons of CYS units in $\beta$ position to amide group (4) and signal around $3.45-3.25$ was related $2 \mathrm{H}$ protons of CYS and $2 \mathrm{H}$ protons of $\mathrm{HE}$ units in $\alpha$ position to amide groups $(3,5)$. Protons at 3.75-3.55 ppm were assigned to $2 \mathrm{H}$ protons of $\mathrm{HE}$ in $\beta$ position to amide group (6). The peak at 4.7 ppm was correlated $1 \mathrm{H}$ proton of methyne group in the backbone (1). It can be seen that the final composition of polyaspartamides

Table 1 Molar ratio of side groups to aspartamide repeating units (NMR) and thiol content of each polymer (Ellman's assay)

\begin{tabular}{lcccccc}
\hline Composition & $\begin{array}{c}\text { Yield } \\
\%\end{array}$ & $\begin{array}{c}\mathrm{X}_{\mathrm{s.g}} \\
\%\end{array}$ & $\begin{array}{c}\mathrm{X}_{\mathrm{CYS}} \\
\%\end{array}$ & $\begin{array}{c}\mathrm{c}_{\mathrm{SH}} \\
\mu \mathrm{mol} / \mathrm{g}\end{array}$ & $\begin{array}{c}\mathrm{X}_{\mathrm{SH}, 0} \\
\%\end{array}$ & $\begin{array}{c}\mathrm{X}_{\mathrm{SH}, \mathrm{tr}} \\
\%\end{array}$ \\
\hline CYS20HE80 & 55 & 82 & 18 & 954 & 18 & 4 \\
CYS20DMP80 & 60 & 78 & 22 & 668 & 13 & 5 \\
CYS20DME80 & 69 & 83 & 17 & 674 & 11 & 2 \\
\hline
\end{tabular}

$\mathrm{X}_{\mathrm{s} . \mathrm{g}}$ : molar percentage of HE, DMP or DME side groups to aspartamide repeating units in $\% ; \mathrm{X}_{\mathrm{CYS}}$ : molar percentage of cysteamine groups to all repeating units in $\%$; $\mathrm{c}_{\mathrm{SH}}$ : thiol content in $\mu \mathrm{mol} / \mathrm{g}$ polymer; $\mathrm{X}_{\mathrm{SH}, 0}$ : molar percentage of thiol groups to all repeating units in $\%$ after preparation; $\mathrm{X}_{\mathrm{SH}, \mathrm{tr}}$ : molar percentage of thiol groups to all repeating units in \% after treatment at $\mathrm{pH}=7.7 ; \quad \mathrm{X}_{\mathrm{s} . \mathrm{g}}$ and $\mathrm{X}_{\mathrm{CYS}}$ were calculated from ${ }^{1} \mathrm{H} \mathrm{NMR}$ spectra (experimental error of determination is approximated as $\pm 5 \%$ ), while $\mathrm{XSH}, 0, \mathrm{X}_{\mathrm{SH}, \mathrm{tr}}$ and $\mathrm{c}_{\mathrm{SH}}$ were determined from Ellman's assay. correlates well with the feed composition in each case, thus all side groups can be attached to polysuccinimide with high conversion (Table 1). The thiol content of polyaspartamides was higher than $10 \%$, and thiol concentration reached a few hundred $\mu \mathrm{mol} / \mathrm{g}$ polymer for each composition.

\subsection{Hydrolytic stability of thiolated polyaspartamides}

Viscosity measurements were carried out to reveal any change in the molecular mass of polyaspartamides treated at $\mathrm{pH}=7.7$. During the viscosity measurements the $\mathrm{pH}$ was kept at 5.5 as polyaspartamides are reported to be stable at acidic pH values [18]. Viscosity values (Fig. 2) confirm that CYS20DME80 went through hydrolytic degradation at $\mathrm{pH}=7.7$ as the viscosity of the polymer solution decreased to almost that of the solvent itself. Only a small reduction of viscosity was observed for CYS20DMP80 and CYS20HE80 indicating that the molecular mass of these polymers did not changed largely. These data suggest that polyaspartamides without DME groups are not prone to main-chain degradation, thus DMP and HE groups can be used later to mitigate the degradation rate of similar derivatives.

${ }^{1} \mathrm{H}$ NMR spectrum of CYS20DME80 before and after treatment was compared to confirm any structural change in addition to the loss of molecular mass (Fig. 3). It can be seen that upon treatment the amount of DME side groups (peak 5) did not change, although a slight downfield shift can be observed for side groups peaks 5 and 7. Cysteamine side groups (peak 3 ) mostly converted into disulfide crosslinks (chemical shift changed from 3.38 to $3.50 \mathrm{ppm}$ ), which clearly supports the theory of main-chain degradation, as

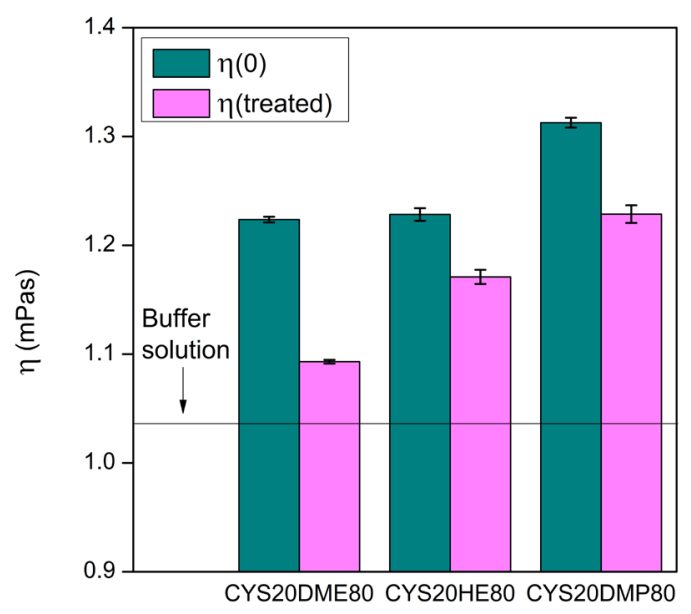

Fig. 2 Viscosity of aqueous solutions of polyaspartamides before and after alkaline treatment, $\eta(0)$ refers to the viscosity of aqueous solution $(\mathrm{pH}=5.5)$ before storage the polymers at $\mathrm{pH}=7.7$, while $\eta$ (treated) to the viscosity after storage the polymers at $\mathrm{pH}=7.7$ 


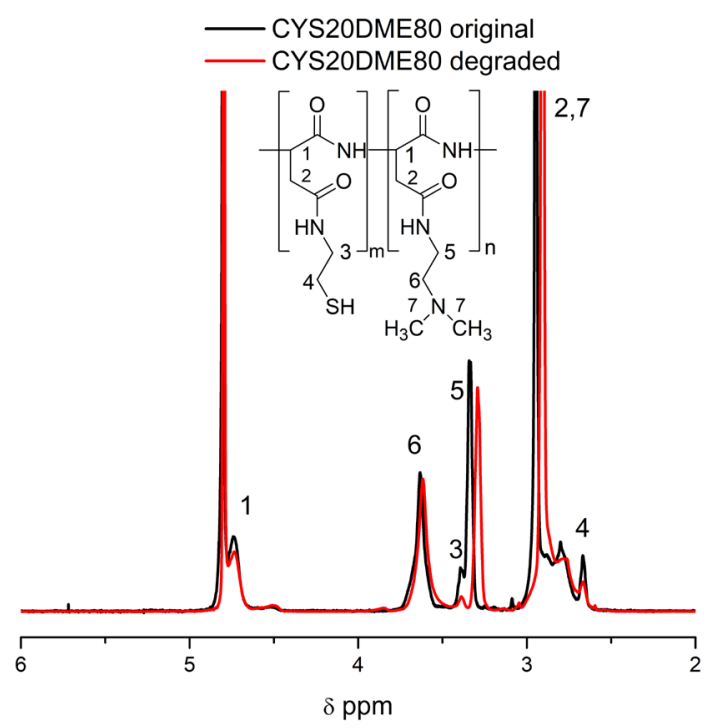

Fig. $3{ }^{1} \mathrm{H}$ NMR spectra of the original and the degraded CYS20DME80

the formation of disulphide linkages would increase considerably the viscosity of the solutions if the main chain remained stable. The formation of disulphide linkages is also proven by the strong reduction of thiol content for all thiolated polyaspartamides (Table 1).

The composition-dependent stability of linear polyaspartamides largely determines the stability of disulfide cross-linked hydrogels.

\subsection{Formation and aqueous stability of disulfide cross- linked polyaspartamide hydrogels}

Polyaspartamides bearing thiol groups are expected to show gelation upon oxidation similarly to thiolated poly(aspartic acid)s [25]. All polyaspartamides studied in this work formed a hydrogel within a few minutes $(5-7 \mathrm{~min})$ after addition of oxidant $(\mathrm{pH}=7.7)$. The gelation process and the stability of the gels were analyzed in detail by oscillatory rheology for CYS20HE80, CYS20DMP80 and CYS20DME80. Small strain oscillatory measurements are extremely useful for following gelation as very broad spectrum of relaxation modes appear during the process and after gelation an extra-long (practically infinite) relaxation mode develops due to the formation of largescale cross-linked structure. To identify the gel point precisely, damping factor ( $\tan \delta=\mathrm{G}^{\prime \prime} / \mathrm{G}^{\prime}$, the ratio of the loss $\left(G^{\prime \prime}\right)$ to the storage $\left(G^{\prime}\right)$ modulus) should be measured over a wide frequency range because it becomes frequency-independent at the gel point. Nevertheless, this type of measurement is usually limited by the long experimental time at low frequencies, thus gelation is often characterized by the recording the $\tan \delta$ values or dynamic moduli over time at a chosen frequency, the latter of which is shown for our samples in Fig. 4. It is worth to note that the measurement could be started only $50 \mathrm{~s}$ after the addition of oxidant, which explains the comparable storage $\left(G^{\prime}\right)$ and loss $\left(G^{\prime \prime}\right)$ modulus at the beginning [30]. The sharp increase of storage modulus indicates the formation of a cross-linked network and the corresponding time interval is the same for CYS20HE80 and CYS20DMP80, while gelation was slower for CYS20DME80. The final value of storage modulus characterizing the strength of the hydrogels formed is also lower for CYS20DME80. Both differences might be explained by the gradual degradation of this polymer even during the gelation process.

Mechanical properties of the same hydrogels were further analyzed by frequency-dependent measurements after gelation (Fig. 5(a, b)). It is known that storage modulus of solid materials is frequency independent over a wide frequency range. CYS20DMP80 and CYS20HE80 hydrogels showed constant storage modulus $\left(G^{\prime}\right)$ almost over the whole frequency range and only a slight increase can be observed at higher frequencies suggesting the appearance of movements with short relaxation times, which is usually assigned to chain entanglements present in all hydrogels (Fig. 5(a)). The loss modulus of these hydrogels was also constant over a wide frequency range showing the absence of any relaxation process. On the contrary, the storage modulus of CYS20DME80 became frequency-dependent at lower frequency and its loss modulus was strongly frequency-dependent suggesting a weaker gel structure. The moduli of CYS20DMP80 and CYS20HE80 remained constant over the duration of the measurements (18 h) while those of CYS20DME80 remarkably

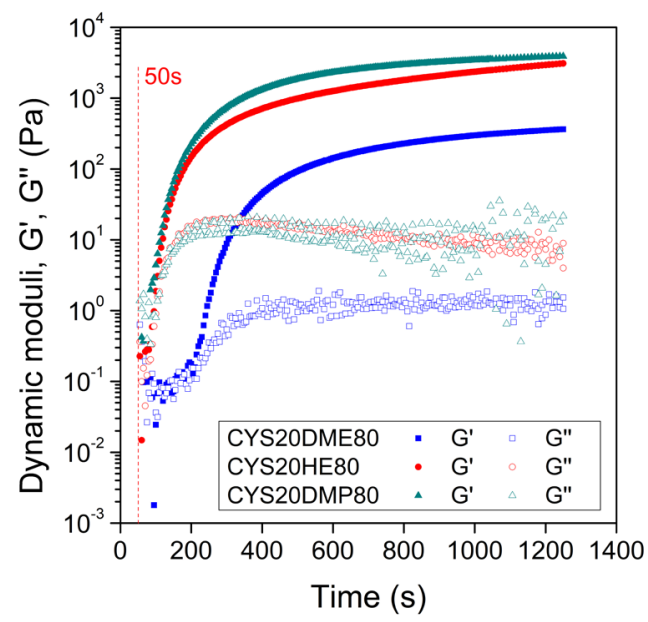

Fig. 4 Time-dependent storage $\left(G^{\prime}\right)$ and loss $\left(G^{\prime \prime}\right)$ modulus of the polyaspartamides during gelation $\left(\gamma=1 \%, \omega=1 \mathrm{rad} \mathrm{s}^{-1}\right)$ 

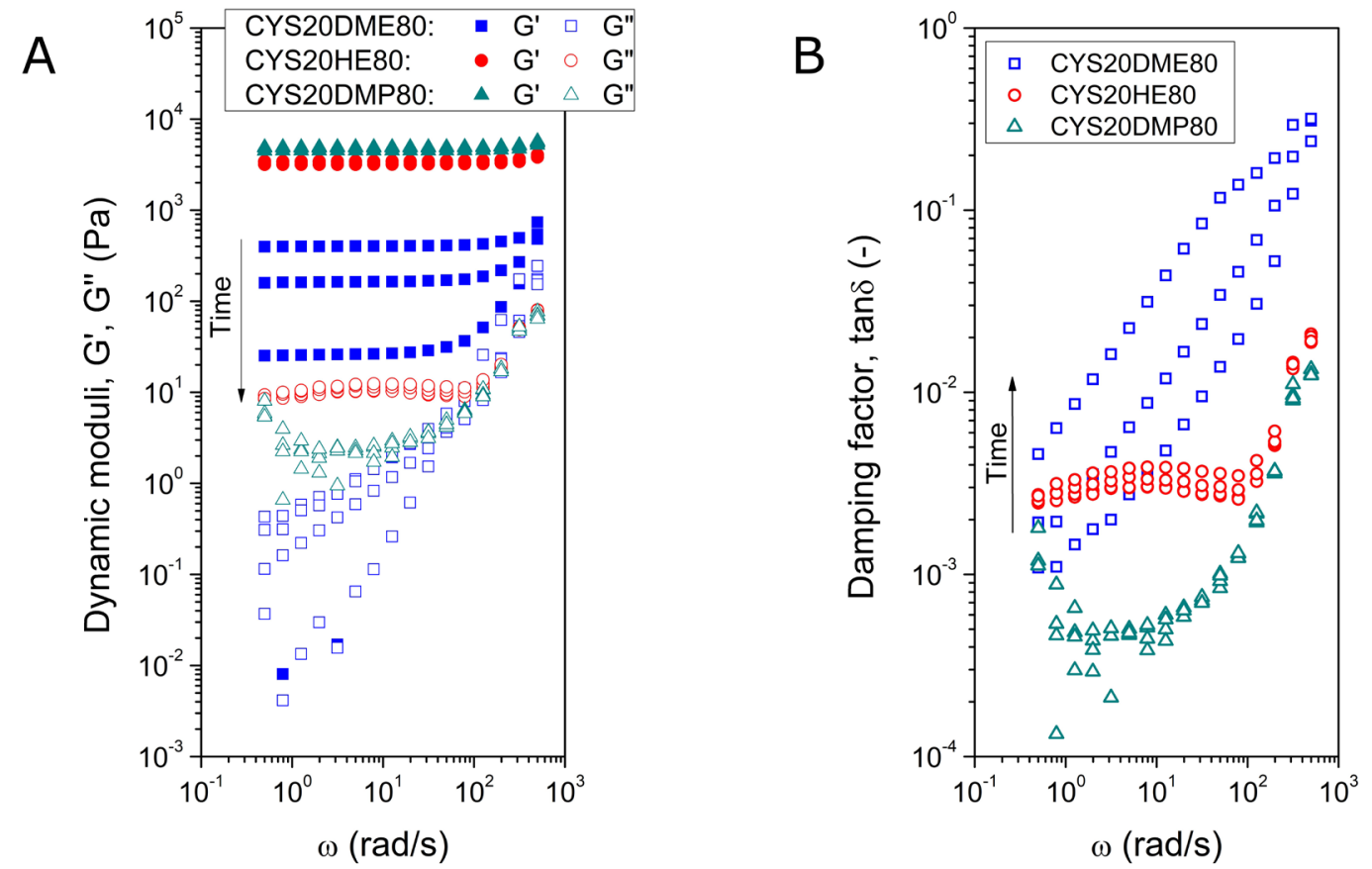

Fig. 5 (a) The frequency-dependence of dynamic moduli 1, 3, 6, $18 \mathrm{~h}$ after gelation; (b) the frequency-dependence of damping factor 1, 3, 6, 18 h after gelation (data points for DME after $18 \mathrm{~h}$ is not shown as damping factor could not be calculated because of the zero value of storage modulus)

decreased. The storage modulus of CYS20DME80 fell by orders of magnitude within a few hours and the range of frequency-independent storage modulus become narrower. Both indicate the weakening of gel structure. Plotting damping factor against frequency demonstrates the differences among the three samples more clearly (Fig. 5(b)). Damping factor of CYS20DMP80 is frequency independent below the threshold value of around $20 \mathrm{rad} / \mathrm{s}$. Similarly, CYS20HE80 showed an increase in damping factor at higher frequencies, but a slight maximum can also be identified at around $6 \mathrm{rad} / \mathrm{s}$. The appearance of the maximum indicates the presence of another relaxation process, possibly connected with larger clusters assembled by second-order interactions. The damping factor of either CYS20HE80 or CYS20DMP80 did not change with time (the measurements were repeated after $3,6,18 \mathrm{~h}$ after gelation) indicating the stability of the gel structure. Contrary to these two polyaspartamides, CYS20DME80 has a strongly frequency-dependent damping factor over the whole experimental range indicating the importance of the viscous character, which can be explained by network imperfections due to the presence of dangling chains with different molecular masses. Furthermore, damping factor quickly increases with time indicating the growth of the viscous character at the expense of the elastic one. Finally the damping factor becomes infinity over the whole frequency range as the storage modulus decreases to zero suggesting the disintegration of the gel structure. This agrees well with the assumption of fast hydrolytic degradation of DME-containing samples, even in their disulfide cross-linked form.

Gel stability was also characterized by recording the plateau modulus (storage modulus determined at the lowest frequency measured) over time. Fig. 6 clearly shows the stability of gels prepared of CYS20HE80 and CYS20DMP80, while the modulus of CYS20DME80 drops to almost zero within a few hours indicating the gel to sol transition. The dissolution of this gel was also observed visually.

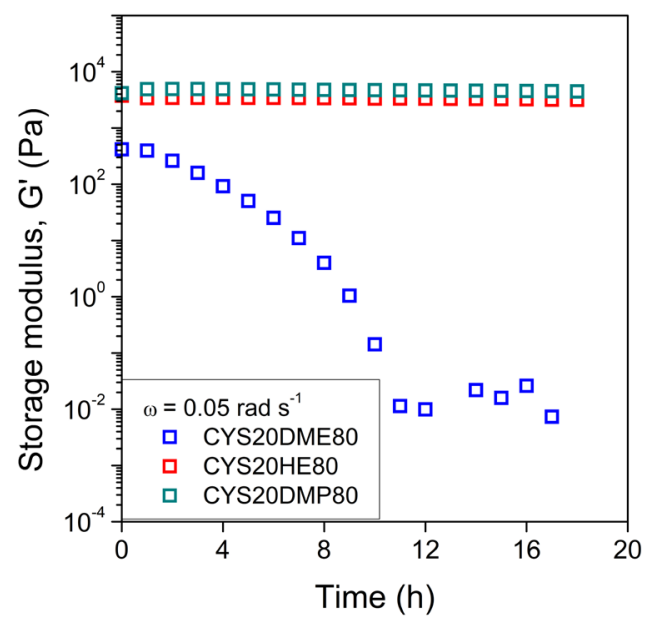

Fig. 6 The change of storage modulus of hydrogels over time $(\omega=0.5 \mathrm{rad} / \mathrm{s})$ 


\section{Conclusion}

The present study showed the successful synthesis of thiolated polyaspartamides with $N, N$-dimethyl-2aminoethyl (DME), N,N-dimethyl-3-aminopropyl (DMP) or 2-hydroxyethyl (HE) side groups. The hydrolytic stability of these polyaspartamides depended on the type of side groups. DME containing polymer showed a loss of molecular mass indicated by viscosity measurements, while polymers with HE and DMP side groups were stable at $\mathrm{pH}=7.7$. Disulfide cross-linked hydrogels were prepared by the oxidation of thiolated polyaspartamides and the stability of hydrogels was investigated by oscillatory rheology. Hydrogels containing HE and DMP groups were stable during the measurements for $18 \mathrm{~h}$, while hydrogel with DME side groups dissolved during measurements within a few hours. The controllable, structure-dependent degradation of polyasparta-

\section{References}

[1] Numata, K. "Poly(amino acid)s/polypeptides as potential functional and structural materials", Polymer Journal, 47, pp. 537-545, 2015. https://doi.org/10.1038/pj.2015.35

[2] Angar, N.-E., Aliouche, D. "Deswelling of Hydrogels in Aqueous and Polyethylene Glycol Solutions. A New Approach for Drug Delivery Application", Periodica Polytechnica Chemical Engineering, 62(2), pp. 137-143, 2018.

https://doi.org/10.3311/PPch.11132

[3] Amri, N., Ghemati, D., Bouguettaya, N., Aliouche, D. "Swelling Kinetics and Rheological Behavior of Chitosan-PVA / Montmorillonite Hybrid Polymers", Periodica Polytechnica Chemical Engineering, 63(1), pp. 179-189, 2019.

https://doi.org/10.3311/PPch.12227

[4] Roweton, S., Huang, S. J., Swift, G. "Poly(aspartic acid): Synthesis, biodegradation, and current applications", Journal of Environmental Polymer Degradation, 5(3), pp. 175-181, 1997. https://doi.org/10.1007/BF02763661

[5] Jalalvandi, E., Shavandi, A. "Polysuccinimide and its derivatives: Degradable and water soluble polymers (review)", European Polymer Journal, 109, pp. 43-54, 2018 https://doi.org/10.1016/j.eurpolymj.2018.08.056

[6] Thombre, S. M., Sarwade, B. D. "Synthesis and Biodegradability of Polyaspartic Acid: A Critical Review", Journal of Macromolecular Science, Part A - Pure and Applied Chemistry, 42(9), pp. 12991315, 2005. https://doi.org/10.1080/10601320500189604

[7] Krisch, E., Gyarmati, B., Szilágyi, A. "Preparation of pH-Responsive Poly(aspartic acid) Nanogels in Inverse Emulsion", Periodica Polytechnica Chemical Engineering, 61(1), pp. 19-26, 2017. https://doi.org/10.3311/PPch.9788

[8] Szilágyi, B. Á., Némethy, Á., Magyar, A., Szabó, I., Bősze, S., Gyarmati, B., Szilágyi, A. "Amino acid based polymer hydrogel with enzymatically degradable cross-links", Reactive Functional Polymers, 133(48), pp. 21-28, 2018.

https://doi.org/10.1016/j.reactfunctpolym.2018.09.015 mides can be useful in the development of degradable drug delivery vehicles.

\section{Acknowledgement}

This research has been supported by the National Research Development and Innovation (NRDI) Fund (TKP2020 IES, Grant No. BME-IE-BIO) based on the charter of bolster issued by the NRDI Office under the auspices of the Ministry for Innovation and Technology, Hungary. Further support was provided by the NRDI Office via grant FK 125074. B. Gyarmati acknowledges the János Bolyai Research Scholarship of the Hungarian Academy of Sciences and the ÚNKP-19-4-BME-421 New National Excellence Program of the Ministry of Human Capacities. A. Mammadova is grateful for the scholarship of Stipendium Hungaricum.

[9] Németh, C., Gyarmati, B., Gacs, J., Salakhieva, D. V., Molnár, K., Abdullin, T., László, K., Szilágyi, A. "Fast dissolving nanofibrous matrices prepared by electrospinning of polyaspartamides", European Polymer Journal, 130, Article No. 109624, 2020. https://doi.org/10.1016/j.eurpolymj.2020.109624

[10] Krisch, E., Gyarmati, B., Barczikai, D., Lapeyre, V., Szilágyi, B. Á., Ravaine, V., Szilágyi, A. "Poly(aspartic acid) hydrogels showing reversible volume change upon redox stimulus", European Polymer Journal, 105, pp. 459-468, 2018. https://doi.org/10.1016/j.eurpolymj.2018.06.011

[11] Park, H. W., Kim, J.-D. "Mucoadhesive interaction of cysteine grafted poly(2-hydroxyethyl aspartamide) with pig mucin layer of surface plasmon resonance biosensor", Journal of Industrial and Engineering Chemistry 15(4), pp. 578-583, 2009. https://doi.org/10.1016/j.jiec.2009.01.016

[12] Uchida, H., Miyata, K., Oba, M., Ishii, T., Suma, T., Itaka, K., Nishiyama, N., Kataoka, K. "Odd-Even Effect of Repeating Aminoethylene Units in the Side Chain of N-Substituted Polyaspartamides on Gene Transfection Profiles", Journal of American Chemical Society, 133(39), pp. 15524-15532, 2011. https://oi.org/10.1021/ja204466y

[13] Miyata, K., Oba, M., Nakanishi, M., Fukushima, S., Yamasaki, Y., Koyama, H., Nishiyama, N., Kataoka, K. "Polyplexes from Poly(aspartamide) Bearing 1,2-Diaminoethane Side Chains Induce pH-Selective, Endosomal Membrane Destabilization with Amplified Transfection and Negligible Cytotoxicity", Journal of American Chemical Society, 130(48), pp. 16287-16294, 2008. https://doi.org/10.1021/ja804561g

[14] Szilágyi, B. Á., Mammadova, A., Gyarmati, B., Szilágyi, A. "Mucoadhesive interactions between synthetic polyaspartamides and porcine gastric mucin on the colloid size scale", Colloids and Surfaces B: Biointerfaces, 194, Article No. 111219, 2020. https://doi.org/10.1016/j.colsurfb.2020.111219 
[15] Samal, S. K., Dash, M., Van Vlierberghe, S., Kaplan, D. L., Chiellini, E., van Blitterswijk, C., Moroni, L., Dubruel, P. "Cationic polymers and their therapeutic potential", Chemical Society Reviews, 41(21), pp. 7147-7194, 2012. https://doi.org/10.1039/c2cs35094g

[16] Yavvari, P. S., Awasthi, A. K., Sharma, A., Bajaj, A., Srivastava, A. "Emerging biomedical applications of polyaspartic acid-derived biodegradable polyelectrolytes and polyelectrolyte complexes", Journal of Materials Chemistry B, 7(13), pp. 2102-2122, 2019. https://doi.org/10.1039/c8tb02962h

[17] Sharma, A., Kundu, S., Reddy M. A., Bajaj, A., Srivastava, A. "Design and Engineering of Disulfide Crosslinked Nanocomplexes of Polyamide Polyelectrolytes: Stability under Biorelevant Conditions and Potent Cellular Internalization of Entrapped Model Peptide", Macromolecular Bioscience, 13(7), pp. 927-937, 2013. https://oi.org/10.1002/mabi.201300018

[18] Kravicz, M. H., Balogh, D. T., Kar, M., Wedepohl, S., Bentley, M. V. L. B., Calderón, M. "Influence of Alkyl Chains of Modified Polysuccinimide-Based Polycationic Polymers on Polyplex Formation and Transfection", Macromolecular Bioscience 19(10), Article No. 1900117, 2019. https://oi.org/10.1002/mabi.201900117

[19] Itaka, K., Ishii, T., Hasegawa, Y., Kataoka, K. "Biodegradable polyamino acid-based polycations as safe and effective gene carrier minimizing cumulative toxicity", Biomaterials, 31(13), pp. 3707-3714, 2010. https://doi.org/10.1016/j.biomaterials.2009.11.072

[20] Lu, Y., Chau, M., Boyle, A. J., Liu, P., Niehoff, A., Weinrich, D., Reilly, R. M., Winnik, M. A. "Effect of Pendant Group Structure on the Hydrolytic Stability of Polyaspartamide Polymers under Physiological Conditions", Biomacromolecules, 13(5), pp. 12961306, 2012. https://doi.org/10.1021/bm2018239

[21] Neuse, E. W., Perlwitz, A. G., Schmitt, S. "Water-soluble polyamides as potential drug carriers. III. Relative main-chain stabilities of side chain-functionalized aspartamide polymers on aqueous-phase dialysis", Die Angewandte Makromolekulare Chemie, 192 (1), pp. 35-50, 1991. https://doi.org/10.1002/apmc.1991.051920103

[22] Wang, X., Ren, H., Yan, Y., Ji, M. "A Novel Bio-based Polyaspartic Acid Copolymer: Synthesis, Structure and Performance of Degradation", Journal of Polymers and the Environment, 26, pp. 4201-4210, 2018.

https://doi.org/10.1007/s10924-018-1293-5
[23] Shinoda, H., Asou, Y., Suetsugu, A., Tanaka, K. "Synthesis and Characterization of Amphiphilic Biodegradable Copolymer, Poly(aspartic acid-co-lactic acid)", Macromolecular Bioscience, 3(1), pp. 34-43, 2003. https://doi.org/10.1002/mabi.200390007

[24] Németh, C., Szabó, D., Gyarmati, B., Gerasimov, A., Varfolomeev, M., Abdullin, T., László, K., Szilágyi, A. "Effect of side groups on the properties of cationic polyaspartamides", European Polymer Journal, 93, pp. 805-814, 2017.

https://doi.org/10.1016/j.eurpolymj.2017.02.024

[25] Gyarmati, B., Vajna, B., Némethy, Á., László, K., Szilágyi, A. "Redox- and pH-Responsive Cysteamine-Modified Poly(aspartic acid) Showing a Reversible Sol-Gel Transition", Macromolecular Bioscience, 13(5), pp. 633-640, 2013. https://doi.org/10.1002/mabi.201200420

[26] Kurakazu, M., Katashima, T., Chijiishi, M., Nishi, K., Akagi, Y., Matsunaga, T., Shibayama, M., Chung, U., Sakai, T. "Evaluation of Gelation Kinetics of Tetra-PEG Gel", Macromolecules, 43(8), pp. 3935-3940, 2010. https://doi.org/10.1021/ma100176f

[27] Krisch, E., Balogh-Weiser, D., Klimkó, J., Gyarmati, B., László, K., Poppe, L., Szilágyi, A. "Composite beads of silica gel, alginate and poly(Aspartic acid) for the immobilization of a lipase enzyme", Express Polymer Letters, 13(6), pp. 512-523, 2019. https://doi.org/10.3144/expresspolymlett.2019.43

[28] Vlasák, J., Rypáček, F., Drobník, J., Saudek, V. "Properties and reactivity of polysuccinimide", Journal of Polymer Science: Polymer Symposia, 66(1), pp. 59-64, 1979. https://doi.org/10.1002/polc.5070660109

[29] Ellman, G. L. "Tissue sulfhydryl groups", Archives of Biochemistry Biophysics, 82(1), pp. 70-77, 1959. https://doi.org/10.1016/0003-9861(59)90090-6

[30] Gyarmati, B., Krisch, E., Szilágyi, A. "In situ oxidation-induced gelation of poly(aspartic acid) thiomers", Reactive and Functional Polymers, 84, pp. 29-36, 2014. https://doi.org/10.1016/j.reactfunctpolym.2014.08.007 